\title{
Scanning method for indoor localization using the RSSI approach
}

\author{
Ahmad Warda ${ }^{1}$, Bojana Petković ${ }^{2}$, and Hannes Toepfer ${ }^{1}$ \\ ${ }^{1}$ Technische Universität Ilmenau, Institute for Information Technology, Ilmenau, Germany \\ ${ }^{2}$ Technische Universität Ilmenau, Institute of Biomedical Engineering and Informatics, Ilmenau, Germany
}

Correspondence to: Ahmad Warda (ahmad.warda@tu-ilmenau.de)

Received: 19 December 2016 - Revised: 10 April 2017 - Accepted: 27 April 2017 - Published: 20 June 2017

\begin{abstract}
This paper presents a scanning method for indoor mobile robot localization using the received signal strength indicator (RSSI) approach. The method eliminates the main drawback of the conventional fingerprint, whose database construction is time-consuming and which needs to be rebuilt every time a change in indoor environment occurs. It directly compares the column vectors of a kernel matrix and signal strength vector using the Euclidean distance as a metric. The highest resolution available in localization using a fingerprint is restricted by a resolution of a set of measurements performed prior to localization. In contrast, resolution using the scanning method can be easily changed using a denser grid of potential sources. Although slightly slower than the trilateration method, the scanning method outperforms it in terms of accuracy, and yields a reconstruction error of only $0.08 \mathrm{~m}$ averaged over 1600 considered source points in a room with dimensions $9.7 \mathrm{~m} \times 4.7 \mathrm{~m} \times 3 \mathrm{~m}$. Its localization time of $0.39 \mathrm{~s}$ makes this method suitable for real-time localization and tracking.
\end{abstract}

\section{Introduction}

Wireless indoor mobile robot localization is a challenging problem and has gained wide attention in the research community (Huang et al., 2016). Location information is essential for the robot to complete the tasks. In many mobile robot applications, especially in the industry, high localization and positioning accuracy are required (Zhang et al., 2014; Röwekämper et al., 2012). Domestic robots and automated guided vehicles (AGV) are examples of mobile robots used inside homes and in the industry, respectively. When a mobile robot does not follow a predefined path, a real-time tracking is necessary. This process can be seen as a sequentially performed localization of a moving robot. Wireless sensor networks (WSNs) are usually used to perform tracking (Zhang et al., 2014) and received signal strength indicator (RSSI) is the most used signal property in wireless indoor localization (Farid et al., 2013). In this paper, we address the problem of indoor mobile robot localization and tracking using WSNs.

Many algorithms exist to estimate a robot location in an indoor environment. Fingerprint is a popular approach used for this purpose. It can provide a highly accurate localization; however, the fingerprint approach requires advanced measurements of RSSI signals associated with the specific points and collected in a database. The size of a database grows if higher resolution is required for a specific application. The closest match between the measurements in the database and the existing measurement is done using deterministic or probabilistic approaches. Improvements in the localization accuracy of the traditional fingerprint technique are achieved by incorporating the weighted fusion procedure (Ma et al., 2015). A fingerprint method using WiFi based on the similarity between unequal length sequences is used by Huang et al. (2016) for localizing a robot. Another frequently used technique to estimate the position of a robot in an indoor environment is trilateration. According to this geometry-based localization technique, the dependency of signal strengths is considered as a function of distance between a transmitter and a receiver. Location estimation can be expressed as the problem of determining the intersection of three spheres by finding a solution for the system of quadratic equations, provided that the centers and radii of spheres are known. Although in the case of precise measurements, trilateration can enable accurate localization, the noisy measurements de- 
grade the quality of trilateration (Yang and Liu, 2010). For improved indoor localization based on trilateration, a technique based on particle filter can be used (Hsu et al., 2016). Finally, the two localization techniques, fingerprint and trilateration, could be integrated (Kodippili and Dias, 2010).

In this paper, we propose a new fast localization scheme based on fingerprint using the RSSI approach. The method has two major contributions to indoor robot localization: first, it does not require offline acquisition, saving a lot of time and manpower, and providing the same accuracy as fingerprint. Second, it outperforms the standard trilateration method, showing lower localization error in a noisy indoor environment. The paper is organized as follows: Sect. 2 describes the problem, defines the forward model, and explains the mathematical background of the method. Section 3 reports the evaluation results of the proposed scanning method with respect to fingerprint and trilateration. Finally, Section 4 concludes the paper and directs future work.

\section{Methodology}

\subsection{Problem description and forward model}

We observed a room with dimensions $9.7 \mathrm{~m} \times 4.7 \mathrm{~m} \times 3 \mathrm{~m}$. In our simulations, we used radio transceiver AT86RF230 from Atmel (2009) with a working frequency of $2.4 \mathrm{GHz}$ (ZigBee/IEEE802.15.4 applications). The transmitter antenna was simulated by the maximum output power of the transmitter of $P_{\mathrm{t}}=+3 \mathrm{dBm}$ with a gain of $G_{\mathrm{t}}=-0.5 \mathrm{dBi}$. The gain of the receiving antenna was also set to $G_{\mathrm{r}}=-0.5 \mathrm{dBi}$. A continuous path of the transmitter was presented by 18 positions equidistantly placed with a step of $0.5 \mathrm{~m}$ at $1 \mathrm{~m}$ height (Fig. 1). We placed three receivers at the ceiling of the room to eliminate obstructions from furniture or other objects. The receivers' positions were given by the following coordinates: $\mathrm{S} 1(4.85 \mathrm{~m}, 0.35 \mathrm{~m}, 3 \mathrm{~m}), \mathrm{S} 2(5.7 \mathrm{~m}, 4.35 \mathrm{~m}, 3 \mathrm{~m})$ and $\mathrm{S} 3(2.95 \mathrm{~m}, 2.35 \mathrm{~m}, 3 \mathrm{~m})$ and shown in Fig. 1 . The received signal power $P_{\mathrm{r}}$ at receiver position $\boldsymbol{R}_{i}$ is related to the power of transmitter $P_{\mathrm{t}}$ at position $\boldsymbol{r}_{j}$ according to the forward model (Eq. 1), assuming a direct path between the receiver and the transmitter and no signal interference occurrence (Garg, 2007):

$P_{\mathrm{r}}=P_{\mathrm{t}}\left(\frac{\lambda}{4 \pi r}\right)^{2} G_{\mathrm{r}} G_{\mathrm{t}}$,

where $r=\left|\boldsymbol{R}_{i}-\boldsymbol{r}_{j}\right|$ represents the distance between the transmitter and the receiver; $\lambda$ is the wavelength of propagation and $G_{\mathrm{r}}$ and $G_{\mathrm{t}}$ are the gains of the receiver and the transmitter antenna, respectively. We composed a kernel matrix of dimensions $n \times m$ according to the forward model (Eq. 1):

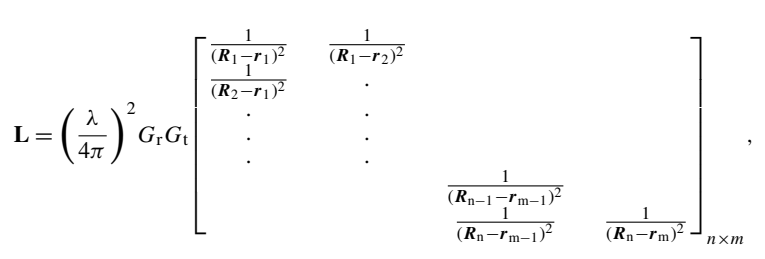

where $n$ corresponds to the number of receivers and $m$ corresponds to the number of discrete source points that the trajectory of the transmitter is modeled with. We assigned to these points the same transmitted power at $m$ discrete moments $\tau_{j}, j=1, \ldots, m$. Then, we introduced a vector of transmitted signal strengths $\boldsymbol{P}_{\mathrm{t}}(\tau)=\left[P_{\mathrm{t} 1}(\tau) P_{\mathrm{t} 2}(\tau) \ldots P_{\mathrm{tm}}(\tau)\right]^{T}$ of dimensions $m \times 1$, where $P_{\mathrm{tj}}=P_{\mathrm{t}} \cdot \delta\left(\tau-\tau_{j}\right), j=1, \ldots, m$. Superscript $T$ indicates the vector transpose. A Dirac delta function is denoted by $\delta\left(\tau-\tau_{j}\right)$, where $j$ indicates a source point where the transmitted antenna is placed along its path at the moment $\tau_{j}$. Now, we can simulate a vector of received power strengths as

$\boldsymbol{P}_{\mathrm{r}}(\tau)=\mathbf{L} \cdot \boldsymbol{P}_{\mathrm{t}}(\tau)$

where $\boldsymbol{P}_{\mathrm{r}}(\tau)=\left[P_{\mathrm{r} 1}(\tau) P_{\mathrm{r} 2}(\tau) \ldots P_{\mathrm{rn}}(\tau)\right]^{T}$ is of dimensions $n \times 1$ and $n$ denotes the number of receivers. We are aware that the model (Eq. 1) and the corresponding kernel matrix (Eq. 2) are, to some degree, imperfect and should be calculated taking into account major obstacles that appear in the room. However, an accurate model requires perfect knowledge of the environment and such a model would lack generality and reusability. For this reason, our approach is presented on a simple RSSI localization model.

\subsection{Scanning method}

First, we introduced discretization over the source space at $1 \mathrm{~m}$ height, defining the total number of possible source points as $M$. The density of the source points depended on the accuracy one wants to achieve. We defined an $M \times$ 1 column vector of transmitted signal strengths $\boldsymbol{P}_{\mathrm{t}}(\tau)=$ $\left[P_{\mathrm{t} 1}(\tau) P_{\mathrm{t} 2}(\tau) \ldots P_{\mathrm{tM}}(\tau)\right]^{T}$ for all discrete moments $\tau=\tau_{j}$, $j=1, \ldots, m$, which models a continuous path of the source antenna. Knowing the receiver position vectors $\boldsymbol{R}_{i}, i=1, \ldots$, $N$, a kernel matrix $\mathbf{L}_{\mathrm{N} \times M}$ is composed according to Eq. (2). The simulated power strength is already obtained using the Eq. (3).

In general, an inverse problem involves estimation of the source vector distribution $\boldsymbol{P}_{\mathrm{t}}(\tau)$ from the measurement vector $\boldsymbol{P}_{\mathrm{r}}(\tau)$. Because we have an isolated source, we are not required to solve the whole system of linear equations and find the distribution of $\boldsymbol{P}_{\mathrm{t}}(\tau)$ over the whole source space. Instead, we can calculate the similarity or distance between the column vectors $\boldsymbol{c}_{j}(\tau), j=1, \ldots, M$ of the kernel matrix $\mathbf{L}_{\mathrm{N} \times \mathrm{M}}$ and the received power strength vector $\boldsymbol{P}_{\mathrm{r}}(\tau)$, for all discrete moments $\tau=\tau_{j}, j=1, \ldots, m$. In the following text, we will skip the notation of the observed moment $\tau$. 


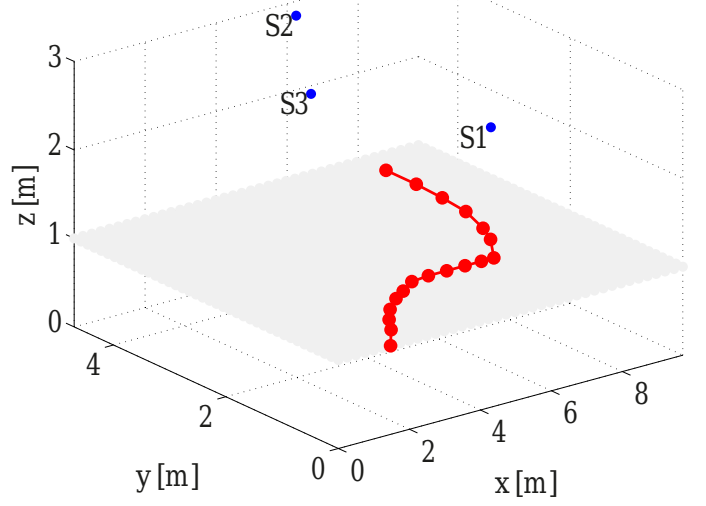

Figure 1. Three receivers are placed at $3 \mathrm{~m}$ height, shown as blue points. The path of the moving transmitter (18 positions) is shown as red points, joined by a red solid line. The plane of the moving transmitter is discretized in $40 \times 40$ source locations shown in grey.
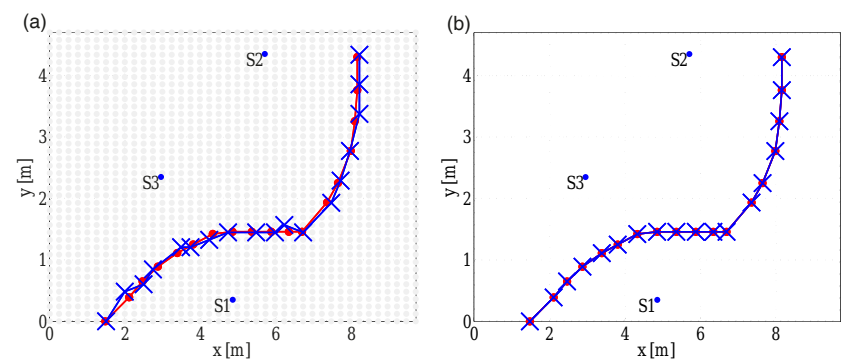

Figure 2. Path reconstruction using the scanning method and fingerprint (a) and trilateration (b) for noise-free simulations.

As a similarity metric between vectors, the cosine between the measurement vector and the columns of a kernel matrix has usually been used (Shi, 2015; Chakraverty, 2014; Berry and Kogan, 2010). However, this measures the similarity of vectors with respect to the origin, and it is not influenced by one magnitude being small compared to the other. This can easily lead to wrong reconstruction because vectors that greatly differ in amplitude can have a very high cosine similarity. Therefore, we used the Euclidean distance $d_{2}\left(\boldsymbol{c}_{j}, \boldsymbol{P}_{\mathrm{r}}\right)$ between the column vectors of a kernel matrix $\boldsymbol{c}_{j}$ and the simulated power strength vector $\boldsymbol{P}_{\mathrm{r}}$

$d_{2}\left(\boldsymbol{c}_{j}, \boldsymbol{P}_{\mathrm{r}}\right)=\left\|\boldsymbol{c}_{j}-\boldsymbol{P}_{\mathrm{r}}\right\|_{2}, j=1, \ldots, M$,

where $\|\cdot\|_{2}$ denotes the Euclidean norm. The location of the source $\hat{j}$ is assigned to the index $j$ corresponding to the minimal Euclidean distance $d_{2}$ to the vector $\boldsymbol{P}_{\mathrm{r}}$, that is,

$\hat{j}=\underset{j}{\arg \min } d_{2}\left(\boldsymbol{c}_{j}, \boldsymbol{P}_{\mathrm{r}}\right)$.

Because we calculated column-by-column the degree of similarity to the measurement vector $\boldsymbol{P}_{\mathrm{r}}$ and in a way scanned the whole source space, we referred to this as a scanning method.
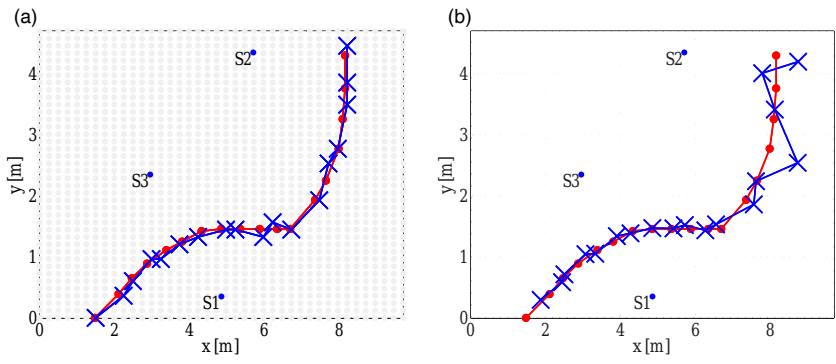

Figure 3. Path reconstruction using the scanning method and fingerprint (a) and trilateration (b) for noisy simulation data.

Table 1. Comparison of reviewed localization methods in terms of time and mean reconstruction error for noise-free simulations.

\begin{tabular}{lrrr}
\hline & $\begin{array}{r}\text { Scanning } \\
\text { method }\end{array}$ & Trilateration & Fingerprint \\
& & & \\
\hline Time per localization (s) & 0.375 & 0.304 & 2.779 \\
MRE (m) & 0.0999 & 0 & 0.0999 \\
\hline
\end{tabular}

\section{Results and discussion}

The quantitative evaluation of the scanning method is performed for the problem setup described in Sect. 2.1 by comparing with the trilateration and fingerprint methods. The methods are compared with respect to the time required for the source localization and the mean reconstruction error (MRE) defined as

$\mathrm{MRE}=\frac{1}{m} \sum_{i=1}^{m}\left|\boldsymbol{r}_{\text {rec }}-\boldsymbol{r}_{\text {real }}\right|$

where $\boldsymbol{r}_{\text {rec }}$ and $\boldsymbol{r}_{\text {real }}$ denote the position vectors of the reconstructed and real simulated sources, respectively. The corresponding simulations have been done for two cases: noisefree simulated data and data with additive white Gaussian noise (AWGN). A discretization of the source space has been made using a step of $0.24 \mathrm{~m}$ in the $x$ direction and $0.12 \mathrm{~m}$ in the $y$ direction, providing $40 \times 40$ considered source locations. Due to a kernel matrix $\mathbf{L}$ of dimensions $3 \times 1600$, our problem is extremely underdetermined. A path of the transmitter is simulated by 18 discrete positions marked by red points connected by a red solid line (Fig. 1).

\subsection{Noise-free simulation}

Comparison of different algorithms is performed on the same noise-free data set. Table 1 shows the time required for one localization and mean reconstruction error MRE averaged over 18 discrete points simulating the path of the source, in the application of the scanning method, trilateration, and fingerprint.

The scanning method localizes a source within $0.375 \mathrm{~s}$, providing a mean reconstruction error of $\mathrm{MRE}=0.099 \mathrm{~m}$. 
Table 2. Comparison of reviewed localization methods in terms of time and mean reconstruction error over 10000 simulation runs under the noisy conditions.

\begin{tabular}{lrrr}
\hline & $\begin{array}{r}\text { Scanning } \\
\text { method }\end{array}$ & Trilateration & Fingerprint \\
\hline Time per localization (s) & 0.396 & 0.344 & 2.920 \\
MRE (m) for & 0.1271 & 0.2255 & 0.1271 \\
10000 iteration runs & & & \\
\hline
\end{tabular}

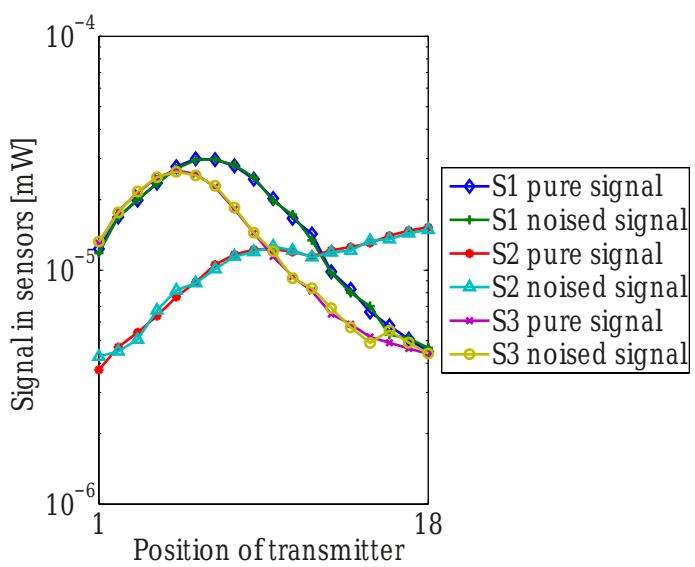

Figure 4. Noise-free signals and signals with additive white Gaussian noise simulated at the positions of three receivers (S1, S2, and S3) and presented in logarithmic scale.

Fingerprint provides the same MRE as the scanning method, requiring, however, much more time, that is, $2.779 \mathrm{~s}$ per one localization. This time consumption is expected because a fingerprint database has to be built in offline mode, prior to localization. Furthermore, a fingerprint database needs to be rescanned and updated, because it is influenced by any change in the environment (e.g., furniture position). In contrast to fingerprint, the scanning method does not require any database and allows for real-time localization. The scanning method can be seen as a kind of fingerprint method with only online mode.

Trilateration performs best in the case of noise-free simulations, accurately localizing the source position within only $0.304 \mathrm{~s}$. However, a noise-free setting is not sufficient because perfect noise-free measurements are never available. Therefore, a noisy system has to be analyzed. A source path reconstructed using the scanning method coincides with the path obtained by fingerprint (Fig. 2a). Source localization using trilateration is shown in Fig. $2 b$.

Note that besides the Euclidean distance (Eq. 5), we have also used a cosine metric as proposed in Zhang et al. (2015) as a similarity metric between the column vectors of a kernel matrix and the simulated power strength vector. However, this metric performed worse than the Euclidean distance and these results are not shown in the paper. The reason is that
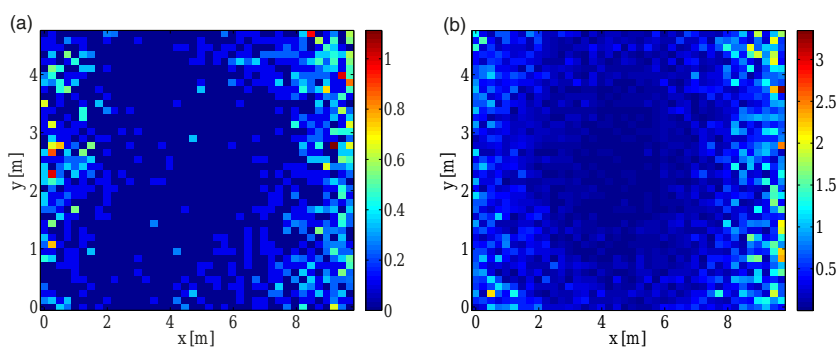

Figure 5. Distribution of the reconstruction error (RE) in the room under noisy conditions and $S N R=103 \mathrm{~dB}$ using the scanning method (a) and trilateration (b). The unit of the color bar is meters.

two vectors can be similar in terms of the angle between them, but they can differ greatly in amplitude. Therefore, the Euclidean distance turned out to be superior over the cosine metric in the application of the scanning method.

\subsection{Simulation with AWGN noise}

To analyze the performance of the scanning method under the effect of noise, we added an additive white Gaussian noise (AWGN) to the simulation data. The signal-to-noise ratio SNR was taken according to the data sheet of transceiver AT86RF230 from Atmel (2009), with RSSI minimal sensitivity of $-91 \mathrm{dBm}$. Considering a transmitted power of $P_{\mathrm{t}}=+3 \mathrm{dBm}$, an SNR value of $103 \mathrm{~dB}$ was used.

Time requirements per one localization and mean reconstruction error applying the scanning method, trilateration, and fingerprint under the same noisy conditions are given in Table 2. Trilateration performed best with respect to the time $(0.344 \mathrm{~s})$ needed for one localization; however, it showed the largest mean reconstruction error of $0.2255 \mathrm{~m}$. Localization accuracy was significantly better in the case of the fingerprint and scanning method, showing the mean value of $0.1271 \mathrm{~m}$ averaged over 18 discrete source positions simulating the trajectory and 10000 iteration runs simulating the data with white Gaussian noise. In terms of time, the scanning method outperformed fingerprint, requiring $0.396 \mathrm{~s}$ per one source localization compared to $2.920 \mathrm{~s}$. This high time consumption makes the fingerprint method inappropriate for real-time target tracking in a dynamical environment like the industry.

Reconstructing paths, relying on noisy data, and applying the scanning method and the fingerprint are shown in Fig. 3a. Figure $3 b$ shows the path reconstruction under the same noise conditions applying the trilateration. One example of noisefree data and data with Gaussian white noise at receiver positions is presented in the logarithmic scale in Fig. 4.

As a final stage, we assessed the robustness of the scanning method through the distribution of the reconstruction (localization) error in the whole room $\mathrm{RE}=\sum_{i=1}^{M}\left|\boldsymbol{r}_{\text {rec }}-\boldsymbol{r}_{\text {real }}\right|$, where $\boldsymbol{r}_{\text {rec }}$ and $\boldsymbol{r}_{\text {real }}$ denote the position vectors of the reconstructed and real simulated sources, respectively. We considered 1600 source points located at the plane of $1 \mathrm{~m}$ height and 
added white Gaussian noise with $\mathrm{SNR}=103 \mathrm{~dB}$. The distributions of the localization error using the scanning method and trilateration are shown in Fig. 5. The unit of the color bar in Fig. 5 is meters. The results in Fig. 5 show that the scanning method exhibits superior performance, and mean localization error is $0.0838 \mathrm{~m}$ over 1600 source points considered in the room, with the maximal error value at $1.113 \mathrm{~m}$. By contrast, the mean reconstruction error using trilateration was $0.3066 \mathrm{~m}$, even showing a maximum of $3.345 \mathrm{~m}$.

The computations were performed with MATLAB2014a on a computer with a CPU (Intel Core i5-3470, 3.2 GHz) and $16 \mathrm{~GB}$ of RAM.

\section{Conclusions}

We studied the problem of wireless indoor mobile robot localization and tracking using noise-free data and data with additive white Gaussian noise at three receiver positions. We proposed a new scanning method to overcome the drawbacks of fingerprint, which includes time-consuming construction of a database and its need for rebuilding every time a significant change in the environment occurs. Moreover, localization in a noisy environment using the scanning method shows a lower error compared to the traditional trilateration. Low computational costs and high accuracy, without the need for a database, make this scanning method a powerful tool for indoor object localization and tracking. Further work will explore 3-D variants of the scanning method using the measured data sets.

Data availability. Data used for transceiver AT86RF230 from Amtel (2009) are available at http://www.atmel.com/images/ doc5131.pdf.

Competing interests. The authors declare that they have no conflict of interest.

Acknowledgements. We would like to thank Roland Eichardt from the Institute of Biomedical Engineering and Informatics at the Technische Universität Ilmenau for the scientific discussions, and also the anonymous reviewers for their critical points, which helped us improve the paper.

Edited by: R. Morello

Reviewed by: two anonymous referees

\section{References}

Atmel: AT86RF230, Atmel, available at: http://www.atmel.com/ images/doc5131.pdf (last access: 28 March 2017), 2009.

Berry, M. W. and Kogan, J.: Text Mining: Applications and Theory, Wiley, 2010.

Chakraverty, S.: Mathematics of Uncertainty Modeling in the Analysis of Engineering and Science Problems, IGI Global, 2014.

Farid, Z., Nordin, R., and Ismail, M.: Recent advances in wireless indoor localization techniques and system, Journal of Computer Networks and Communications, 2013.

Garg, V. K.: Wireless Communications \& Networking, Elsevier Morgan Kaufmann, 2007.

Hsu, C.-C., Yeh, S.-S., and Hsu, P.-L.: Particle filter design for mobile robot localization based on received signal strength indicator, T. I. Meas. Control, 38, 1311-1319, https://doi.org/10.1177/0142331215586615, 2016.

Huang, X., Fan, J., Wu, M., and Gu, Y.: Indoor Mobile Robot Positioning Based on Wireless Fingerprint Matching, World Academy of Science, Engineering and Technology, International Journal of Computer, Electrical, Automation, Control and Information Engineering, 10, 1198-1202, 2016.

Kodippili, N. S. and Dias, D.: Integration of fingerprinting and trilateration techniques for improved indoor localization, in: 2010 Seventh International Conference on Wireless and Optical Communications Networks - (WOCN), 1-6, https://doi.org/10.1109/WOCN.2010.5587342, 2010.

Ma, R., Guo, Q., Hu, C., and Xue, J.: An improved WiFi indoor positioning algorithm by weighted fusion, Sensors, 15, 2182421843, 2015.

Röwekämper, J., Sprunk, C., Tipaldi, G. D., Stachniss, C., Pfaff, P., and Burgard, W.: On the position accuracy of mobile robot localization based on particle filters combined with scan matching, in: 2012 IEEE/RSJ International Conference on Intelligent Robots and Systems, 3158-3164, https://doi.org/10.1109/IROS.2012.6385988, 2012.

Shi, Y.: Emerging Research on Swarm Intelligence and Algorithm Optimization, Hershey: IGI Global, 2015.

Yang, Z. and Liu, Y.: Quality of Trilateration: Confidence-Based Iterative Localization, IEEE T. Parall. Distr., 21, 631-640, https://doi.org/10.1109/TPDS.2009.90, 2010.

Zhang, A., Yuan, Y., Wu, Q., Zhu, S., and Deng, J.: Wireless Localization Based on RSSI Fingerprint Feature Vector, Int. J. Distrib. Sens. N., 11, 528-747, https://doi.org/10.1155/2015/528747, 2015.

Zhang, L., Lim, C.-C., Chen, Y., and Karimi, H. R.: Tracking mobile robot in indoor wireless sensor networks, Mathematical Problems in Engineering, 2014. 